\title{
Welcher Weg? A trajectory representation of a quantum Young's diffraction experiment
}

\author{
Edward R. Floyd \\ 10 Jamaica Village Road, Coronado, CA 92118-3208, USA \\ floyd@mailaps.org
}

30 July 2007

\begin{abstract}
The double slit problem is idealized by simplifying each slit by a point source. A composite reduced action for the two correlated point sources is developed. Contours of the reduced action, trajectories and loci of transit times are developed in the region near the two point sources. The trajectory through any point in Euclidian 3-space also passes simultaneously through both point sources.
\end{abstract}

PACS Nos. 3.65Ta, 3.65Ca, 3.65Ud

Keywords: interference, Young's experiment, trajectory representation, entanglement, nonlocality, determinism

Short running title: Welcher Weg

\section{INTRODUCTION}

One of the first examples of wave-particle duality in quantum mechanics is the double slit experiment which exhibits the wave and the particle properties. The wave properties are exhibited by diffraction patterns analogous to Young's optical experiment while a detector still registers individual particles whose spatial distributions are consistent with Young's diffraction. Attempts at detecting through which slit the particle has passed destroys the interference between the two slits.

The trajectory representation has been developed as a deterministic theory of quantum mechanics. 1- 6 , Faraggi and Matone have shown that the foundations of quantum mechanics can be developed from the quantum equivalence principle, which is consistent with the trajectory theory, without any of the philosophy of the Copenhagen interpretation. 4 The welcher Weg aspect of the quantum Young's diffraction experiment is now ripe for an investigation using the deterministic trajectory representation of quantum mechanics.

The particular form, used herein, of the quantum Young's diffraction experiment examines interference between a pair of coherent secondary point source activated coherently by a primary source. Substituting secondary point sources for the traditional slits simplifies the mathematics, which can be done in closed form, without any loss of welcher Weg physics. Each secondary point source emits a spherical wave that is a component of the total wave function for a solitary diffracted quantum particle. By themselves, the spherical wave components do not represent particular states in the deterministic trajectory representation. The total wave function is shown to be a dispherical wave function for the self-entangled quantum particle that is synthesized from the pair of individual spherical waves coherently emitted by the pair of secondary point sources. The terminology "self-entangled" emphasizes that dispherical wave function may represent a solitary quantum particle whose components, the pair of spherical waves emitted by the coherent pair of secondary point sources, are entangled. A self-entangled wave function for a quantum particle implies a solitary self-entangled particle and is distinguished from an entangled wave function for an entangled pair of 
quantum particles. This investigation develops from the synthesized dispherical wave function the reduced action (Hamilton's characteristic function) that is a generator of the motion for a solitary self-entangled quantum particle. Subsequently the investigation, by applying Jacobi's theorem to the reduced action, develops the trajectory and motion for a solitary self-entangled quantum particle to resolve welcher Weg. This procedure is replicated to establish various other trajectories using different constants of the motion. Both cylindrical and prolate spheroidal coordinate systems are used in this exposition for computational flexibility, as a computational check, and to gain insight. The cylindrical coordinates are more familiar and more closely related to the traditional presentation of the double slit experiment in the Fraunhofer region. The prolate spheroidal coordinate system is the natural coordinate system for investigating twocenter phenomenon. Consequently, the results herein are presented mostly in prolate spheroidal coordinates for heuristic purposes and to exhibit insight. 7

The thrust of this investigation is to determine through which secondary point source the trajectory passes. The investigation therefore concentrates mainly in the region near the pair of secondary point sources. This region is well within the region of Fresnel diffraction. However, Fresnel approximations are not needed herein as the diffraction for a pair of secondary point sources can be determined exactly in closed form. All trajectories for the solitary self-entangled quantum particle are shown to be strongly nonlocal as each individual trajectory is shown to pass simultaneously through both secondary point sources through a series of the trajectory segments that alternate forward and retrograde motion with respect to time. The nonlocality of the dispherical particle implies that that the trajectory is for a distributed particle rather than a point particle.

Manifestation of interference effects including reinforcement and destruction is beyond the scope of this welcher Weg investigation. A companion article shows how the deterministic trajectory representation exhibits interference effects between plane wave functions that is consistent with the quantum equivalence principle of Faraggi and Matone 4 and does not resort to Born's probability density of the Copenhagen interpretation. 3

Philippidis, Dewdney and Hiley have developed Bohmian trajectories for the double slit experiment. 8 Guantes, Sanz, Margalef-Roig and Miret-Artés have revisited the double slit experiment to develop Bohmian trajectories, classical trajectories and the standard (wave function) quantum representation for a "softwalled" double slit barrier. 9) However, Bohmian mechanics 10 and the trajectory representation have different equations of motion. 2/4|6]11 . Consequently the trajectories of the two representations are different and imply different physics and philosophy as one can learn by comparing the findings of these investigations.

In Sect. 2, the wave function, generator of the motion (reduced action), trajectory equation and equation of motion are developed for the solitary self-entangled quantum particle of the quantum Young's diffraction experiment. In Sect. 3, examples of the contours of reduce action, trajectories and loci of transit times are exhibited and discussed for the solitary self-entangled quantum particle. In Sect. 4, the trajectory for the solitary self-entangled quantum particle is shown to transit simultaneously both coherent secondary point sources resolving welcher Weg. In the Appendix, the experiment is modified to render a set of self-entangled wave functions that are shown to synthesize spherical waves.

\section{FORMULATION}

The formulation will be developed in both a modified prolate spheroidal coordinate system $(\eta, \xi, \phi)$ and in the more familiar cylindrical coordinate system $(\rho, z, \phi)$ to facilitate insight, accessibility and computational flexibility. Computations were conducted in both coordinate systems, but the results will be presented for heuristic purposes mostly in the modified prolate spheroidal coordinate system with scale factors (metrical coefficients) modified by Morse and Feshbach. 7 The two foci of the set of nested spheroids are at $(\rho, z, \phi)=$ $(0, \pm a / 2,-\pi \leq \phi \leq \pi)$ in cylindrical coordinates. The distances from the foci to a point $(\rho, z, \phi)$ are given in cylindrical and prolate spheroidal coordinates by 7

$$
r_{1}=\left[\rho^{2}+(z+a / 2)^{2}\right]^{1 / 2}=a(\xi+\eta) / 2 \text { and } r_{2}=\left[\rho^{2}+(z-a / 2)^{2}\right]^{1 / 2}=a(\xi-\eta) / 2 .
$$


The modified prolate spheroidal coordinate system $(\xi, \eta, \phi)$, where $\xi$ is the ellipsoidal coordinate, $\eta$ is the hyperboloidal coordinate, and $\phi$ is the azimuthal coordinate, is specified by

$$
\xi=\left(r_{1}+r_{2}\right) / a, \quad \eta=\left(r_{1}-r_{2}\right) / a \text { and } \phi=\arctan (y / x) .
$$

For the modified scale factors, see Morse and Feshbach. 7 The foci of the spheroids are at $(\xi, \eta, \phi)=$ $(1, \pm 1,-\pi \leq \phi \leq \pi)$ in prolate spheroidal coordinates. The line $\xi=1$ lie on the principal axis of the set of nested prolate spheroids.

Let us consider two secondary point sources displaced from each other by the distance $a$ where each secondary source specifies one of the foci for an infinite set of spheroids. The secondary point sources are coherently actuated with equal strength by a sole primary source so that the secondary sources emit simultaneously components of equal magnitude of a solitary spinless quantum particle of mass $m$ and energy $\hbar^{2} k^{2} /(2 m)$. Each of the two secondary point sources, if acting alone, would be the source for a stationary spherical wave function given by

$$
\psi_{1}=\frac{\exp \left(i \boldsymbol{k}_{1} \cdot \boldsymbol{r}_{1}\right)}{r_{1}}=\frac{\exp \left(i k r_{1}\right)}{r_{1}}
$$

and

$$
\psi_{2}=\frac{\exp \left(i \boldsymbol{k}_{2} \cdot \boldsymbol{r}_{2}\right)}{r_{2}}=\frac{\exp \left(i k r_{2}\right)}{r_{2}}
$$

where $\boldsymbol{k}_{i}$ is colinear with $\boldsymbol{r}_{i}$ and $\boldsymbol{k}_{1} \cdot \boldsymbol{k}_{1}=\boldsymbol{k}_{2} \cdot \boldsymbol{k}_{2}=k^{2}$. The two point secondary source problem is azimuthally invariant. A dispherical wave function, $\psi_{d}$ can be synthesized from its two components, $\psi_{1}$ and $\psi_{2}$, by

$\psi_{d}=\psi_{1}+\psi_{2}=\left[r_{1}^{-2}+r_{2}^{-2}+2 r_{1}^{-1} r_{2}^{-1} \cos \left(\boldsymbol{k}_{1} \cdot \boldsymbol{r}_{1}-\boldsymbol{k}_{2} \cdot \boldsymbol{r}_{2}\right)\right]^{1 / 2} \exp \left[i \arctan \left(\frac{r_{2} \sin \left(\boldsymbol{k}_{1} \cdot \boldsymbol{r}_{1}\right)+r_{1} \sin \left(\boldsymbol{k}_{2} \cdot \boldsymbol{r}_{2}\right)}{r_{2} \cos \left(\boldsymbol{k}_{1} \cdot \boldsymbol{r}_{1}\right)+r_{1} \cos \left(\boldsymbol{k}_{2} \cdot \boldsymbol{r}_{2}\right)}\right)\right]$.

As $\psi_{d}$ is not factorable into a product of $\psi_{1}$ and $\psi_{2}$, the two components, $\psi_{1}$ and $\psi_{2}$, are entangled in $\psi_{d}$. The quantum particle that is emitted from the two secondary point sources has $\psi_{d}$ as its wave function and is self-entangled. In this investigation, which examines the behavior of $\psi_{d}$ as a solitary particle, the components $\psi_{1}$ and $\psi_{2}$ do not represent a pair of particles that entangle with each other. For completeness, the cosine term in the amplitude for $\psi_{d}$ in Eq. (3) manifests interference.

A generator of the motion, the reduced action (Hamilton's characteristic function), $W_{d}$, for the selfentangled wave function can be extracted from Eq. (3) as 3

$$
\begin{aligned}
W_{d} & =\hbar \arctan \left(\frac{r_{2} \sin \left(\boldsymbol{k}_{1} \cdot \boldsymbol{r}_{1}\right)+r_{1} \sin \left(\boldsymbol{k}_{2} \cdot \boldsymbol{r}_{2}\right)}{r_{2} \cos \left(\boldsymbol{k}_{1} \cdot \boldsymbol{r}_{1}\right)+r_{1} \cos \left(\boldsymbol{k}_{2} \cdot \boldsymbol{r}_{2}\right)}\right) \\
& =\hbar \arctan \left(\frac{\left[\rho^{2}+(z-a / 2)^{2}\right]^{1 / 2} \sin \left[k_{\rho} \rho+k_{z}(z+a / 2)\right]+\left[\rho^{2}+(z+a / 2)^{2}\right]^{1 / 2} \sin \left[k_{\rho} \rho+k_{z}(z-a / 2)\right]}{\left[\rho^{2}+(z-a / 2)^{2}\right]^{1 / 2} \cos \left[k_{\rho} \rho+k_{z}(z+a / 2)\right]+\left[\rho^{2}+(z+a / 2)^{2}\right]^{1 / 2} \cos \left[k_{\rho} \rho+k_{z}(z-a / 2)\right]}\right) \\
& =\hbar \arctan \left(\frac{(\xi-\eta) \sin [k(\xi+\eta) a / 2]+(\xi+\eta) \sin [k(\xi-\eta) a / 2]}{(\xi-\eta) \cos [k(\xi+\eta) a / 2]+(\xi+\eta) \cos [k(\xi-\eta) a / 2]}\right) .
\end{aligned}
$$

This generator of the motion, $W_{d}$, for the dispherical particle of quantum Young's diffraction experiment is in Euclidean 3-space and not in Hilbert space. Faraggi and Matone have shown that the reduced action may be derived by the quantum equivalence principle independent of the Schrödinger equation. 4. The reduced action for the self-entangled wave function is independent of $\phi$, which manifests azimuthal symmetry. By Eq. (4), $W_{d}$, albeit independent of $\phi$, does not contain a cyclic coordinate. Neither is it further separable. 
In the vicinity near one of the secondary point source by Eq. (44), the degree that $W_{d}$ mimics reduced action of that secondary point source being the sole point source increases with nearness to that point source.

The trajectory equation for the self-entangled wave function can be developed from the reduced action by Jacobi's theorem $\beta_{i}=\partial W_{d} / \partial \alpha_{i}$ where $\alpha_{i}$ is one of the independent constants of integration and $\beta_{i}$ is its associated constant coordinate. This procedure here differs with Bohmian mechanics, which does not employ Jacobi's theorem. 10 Following Goldstein 12 one may choose other independent quantities, $\gamma_{i}$ 's where each $\gamma_{i}$ is a function of all the $\alpha_{i}$ 's. The $\gamma_{i}$ 's are constant momenta albeit not necessarily the integration constants that arise by integrating the Hamilton-Jacobi equation. Jacobi's theorem still holds for the $\gamma_{i}$ 's. Here, $\hbar k_{z}$ has been selected for the representation in cylindrical coordinates to be a constant momenta. The trajectory equation for $\psi_{d}$ is rendered in the $\rho, z$-plane by Jacobi's theorem as

$$
\beta_{z}=\hbar^{-1} \frac{\partial W_{d}}{\partial k_{z}}=\frac{r_{2}^{2}\left[(z+a / 2)-\frac{k_{z}}{k_{\rho}} \rho\right]+r_{1}^{2}\left[(z-a / 2)-\frac{k_{z}}{k_{\rho}} \rho\right]+2 r_{1} r_{2} \cos \left[k\left(r_{1}-r_{2}\right)\right]\left[z-\frac{k_{z}}{k_{\rho}} \rho\right]}{\left[r_{2} \cos \left(k r_{1}\right)+r_{1} \cos \left(k r_{2}\right)\right]^{2}+\left[r_{2} \sin \left(k r_{1}\right)+r_{1} \sin \left(k r_{2}\right)\right]^{2}}
$$

where for compactness and didactic purposes not all the distances, $r_{1}$ and $r_{2}$, have not been expanded and where $k_{\rho}$ is another $\gamma$ given by $k_{\rho}=+\left(k^{2}-k_{z}^{2}\right)^{1 / 2}$ where the sign of $k_{\rho}$ is positive for outgoing radiation. We assume that the trajectory originates at the secondary point source at the upper focus. Hence, for $r_{2}=0, \rho=0$ and $z=a / 2$, then $\beta_{z}=0$. This permits us to simplify Eq. (5) by

$$
r_{2}^{2} \underbrace{\left[(z+a / 2)-\frac{k_{z}}{k_{\rho}} \rho\right]}_{\text {lower source alone }}+r_{1}^{2} \underbrace{\left[(z-a / 2)-\frac{k_{z}}{k_{\rho}} \rho\right]}_{\text {upper source alone }}+2 r_{1} r_{2} \underbrace{\cos \left[k\left(r_{1}-r_{2}\right)\right]\left(z-\frac{k_{z}}{k_{\rho}} \rho\right)}_{\text {interference effects }}=0 .
$$

Equation (6) has been organized into the weighted contributions (sub-trajectories) from individual secondary sources acting alone and from interference effects. The weighting of an individual contribution from a secondary source acting alone is proportional to the square of the distance from the alternate secondary source while the weighting of the contribution from the interference effects is proportional to twice the product of the two distances from the individual secondary sources. We note that these three sub-trajectories have the same constant of the motion, $\eta_{a}$. The corresponding trajectory equation equation in prolate spheroidal coordinates may be expressed as

$$
\begin{gathered}
(\xi-\eta)^{2}\left\{\eta_{a}\left[\left(\xi^{2}-1\right)\left(1-\eta^{2}\right)\right]^{1 / 2}-\left(1-\eta_{a}^{2}\right)^{1 / 2}(\xi \eta+1)\right\} \\
+(\xi+\eta)^{2}\left\{\eta_{a}\left[\left(\xi^{2}-1\right)\left(1-\eta^{2}\right)\right]^{1 / 2}-\left(1-\eta_{a}^{2}\right)^{1 / 2}(\xi \eta-1)\right. \\
+2\left(\xi^{2}-\eta^{2}\right) \cos (k a \eta)\left\{\eta_{a}\left[\left(\xi^{2}-1\right)\left(1-\eta^{2}\right)\right]^{1 / 2}-\left(1-\eta_{a}^{2}\right)^{1 / 2} \xi \eta\right\}=0
\end{gathered}
$$

where $\eta_{a}$ is the constant of the motion and is the $\eta$-asymptote of the trajectory as it propagates without bound. The top, middle and bottom lines on the left side of Eq. (7) represent in prolate spherical coordinates the contributions due to the lower secondary source alone, upper secondary source alone and interference effects respectively. The trajectory equations are implicit functions. Equation (6) renders $(\rho, z)$ mutually implicit while Eq. (7) renders $(\xi, \eta)$ mutually implicit.

There is a fundamental simplicity that has been achieved by establishing the trajectory of the quantum dispherical particle. Only one constant of the motion is needed to establish the trajectory even though the equation of motion may exhibit vestiges of motion of its components and the interference between its components as explicitly shown by Eq. (6). Otherwise, had one worked directly with trajectories for the component wave functions $\psi_{1}$ and $\psi_{2}$, then the worker would need two constants of the motion to describe two trajectories (for $\psi_{1}$ and $\psi_{2}$ ) for the motion of the dispherical particle.

Note also that the trajectories determined by Jacobi's theorem are not necessarily orthogonal to the contours of reduced action. $3[11$

For completeness, had we been investigating the entangled motion of two particles where each secondary source had simultaneously emitted an identical particle, then we would have proceeded as before and synthesized the entangled wave function, and established the reduced action for the entangled pair. The trajectory 
for the entangled pair would be specified by a single constant of the motion determined by Jacobi's theorem. Note that this procedure would render the trajectory of the entangled pair as a whole and not the trajectory of one particle of the entangled pair under a quantum pressure due to a Bohmian quantum potential for the pair of identical particles. One can generalize for entangled ensembles of $N$ particles. By extending a procedure given by Bohm, 10 one can synthesize an entangled wave function, $\psi_{\mathcal{E}}$, from the ensemble of wave functions, $\psi_{j}, j=1,2, \cdots, N$, where each wave function represents one of entangled $N$ particles, by

$$
\psi_{\mathcal{E}}=\left(\mathcal{X}^{2}+\mathcal{Y}^{2}\right)^{1 / 2} \exp [i \hbar \arctan (\mathcal{Y} / \mathcal{X})]
$$

with

$$
\mathcal{X}=\Re\left[\sum_{j=1}^{N} \psi_{j}\right]=\sum_{j=1}^{N} \Re\left[\psi_{j}\right] \text { and } \mathcal{Y}=\Im\left[\sum_{j=1}^{N} \psi_{j}\right]=\sum_{j=1}^{N} \Im\left[\psi_{j}\right]
$$

While this investigation to resolve welcher Weg concentrates upon the Fresnel region ( $1 \leq \xi<6$ herein), let us now briefly examine qualitatively the trajectories in the Fraunhofer region. In the limit $\xi \rightarrow \infty$, then by Eqs. (6) and (77) $z / \rho \rightarrow k_{z} / k_{\rho}$ rendering the expected behavior of the trajectory in the infinitely outer region. Hence, $k_{\rho}$ and $k_{z}$ may be identified with the asymptotic direction of the trajectory. In prolate spheroidal coordinates, the trajectory and the hyperboloid of revolution specified by $\eta_{a}$ have the common asymptote which is a cone whose generating line from the origin has the angle $\theta$ with the $z$-axis or the ellipsoidal principal axis where $\theta$ is given, as expected, by

$$
\theta=\arccos \left(\eta_{a}\right)=\arctan \left(k_{\rho} / k_{z}\right) .
$$

The concept that $\psi_{d}$ is synthesized from $\psi_{1}$ and $\psi_{2}$ follows from the superpositional principal for linear homogeneous differential equations. In the Appendix, a modified experiment is described where $\psi_{1}$ and $\psi_{2}$ are synthesized from two different dispherical wave functions.

The equation of motion for the dispherical wave function is rendered by Jacobi's theorem, $t-\tau=\partial W_{d} / \partial E$ where $\tau$ specifies the epoch. Jacobi's theorem gives

$$
t-\tau=\left(1-\frac{2}{\xi^{2}+\eta^{2}+\left(\xi^{2}-\eta^{2}\right) \cos (k \eta a)}\right) \frac{m \xi \eta a}{\hbar k \eta_{a}}
$$

for motion projected across the $\eta$-coordinates. The equation of motion must be consistent with the side relation that all points $(\xi, \eta)$ obey the trajectory equation, Eq. (7), with the constant of the motion $\eta_{a}$. For $\eta_{a} \rightarrow 0$, Eq. (8) becomes singular and may be replaced using Eq. (7) by an alternative form

$$
t-\tau=\frac{m \eta a}{\hbar k\left(1-\eta_{a}^{2}\right)^{1 / 2}}
$$

for motion projected across $\xi$-coordinates.

\section{APPLICATION}

\subsection{Reduced Action}

Let us now consider an example $m=1, \hbar=1, a=1$, and $k=15.2$. The value of $k$ was chosen so that $k a / \pi$ would not be a rational number for greater generality. The contours for reduced action for the dichromatic particle are determined by Eq. (4) for $W=0.5 h, 1 h, 1.5 h, \cdots, 5 h$ and exhibited in the $\xi, \eta$-plane on Fig. 1. By symmetry, Fig. 1 need only only cover the range $0 \leq \eta \leq 1$ as there exists a mirror symmetry on the plane $\eta=0$ in addition to the azimuthal symmetry in $\phi$. The contours of constant $W_{d}$ orthogonally intersect the plane $\eta=0$ manifesting the mirror symmetry and orthogonally intersect the axis $\xi=1$ or $\eta=1$ manifesting azimuthal symmetry. The implicit relationships between $\eta$ and $\xi$ or $z$ and $\rho$ were established by 
solving Eq. (44) numerically by the secant method. The contours of reduced action manifest self interference on Fig. 1 as wrinkles in the contours reminiscent of the serpentine contours exhibited for interfering plane

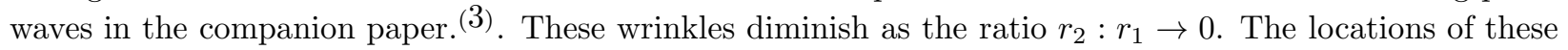
wrinkles are shown by Fig. 2 to occur near $k a \eta=(2 n-1) \pi, n=1,2, \cdots$ in general or, for $k=15.2$ and $a=1$, in the vicinity of the hyperboloids $\eta=0.207,0.620$ near where maximum destructive interference occurs between $\psi_{1}$ and $\psi_{2}$. The distribution of the wrinkles in $W_{d}$ with regard to ka $\eta$ can be substantiated be considering Fig. 3 which exhibits the contours for $W_{d}$ for $m=1, \hbar=1, a=1$, and $k=24.3$. This change in $k$ induces four wrinkles in Fig. 3 in the vicinity of the hyperboloids $\eta=0.129,0.388,0.646,0.905$ where maximum destructive interference occurs.

The behavior of the contour of $W_{d}$ at the origin $(\xi, \eta)=(1,0)$ is interesting and exhibited in Fig. 4. Figure 4 exhibits the behavior of three contours separated by $0.001 \mathrm{~h}$ in action in the vicinity of the origin. Figure 4 is also presented in cylindrical coordinates as prolate spheroidal coordinates do not render any physical insight at the fine scale used therein. Contours of constant reduced action less than approximately $W_{d}=1.316815$ are disjointed with two sets of contours: each enclosing one or the two secondary point sources. At approximately $W_{d}=1.316815$, the two disjointed contours of reduced action merge at the origin. At the origin, the contour must instantaneously transition from orthogonal to the ellipsoidal principal axis $(1, \epsilon)$ to orthogonal to the plane $(1+\epsilon, 0)$ in the limit $\epsilon \rightarrow 0$. Likewise a similar situation is happening for the mirror-symmetric partner contour orthogonal to the ellipsoidal principal axis $(1,-\epsilon)$. As $\epsilon \rightarrow 0$ from above, the radii of curvature of the two mirror-symmetric partner contours are vertically aligned and go to zero from opposite signs, the mirror-symmetric partner contours become tangent albeit the symmetric partner contours have infinite curvature of opposite signs inducing a zero degree of osculation, and their evolutes merge vertically at $(1,0)$. At and only at $\epsilon=0$, the infinite curvatures of opposite sign at the point of tangency $(1,0)$ of the two mirror-symmetric contours form a third contour from the two mirror-symmetric partner contours. This third contour has infinite curvature at $(1,0)$ but whose radius of curvature while zero is, in the limit $\epsilon \rightarrow 0$, horizontally aligned, which allows the two mirror symmetric partner contours to be joined at $\epsilon=0$ to form a single contour. Succeeding contours of larger action higher will be orthogonal to the plane $\eta=0$.

\section{$3.2 \quad$ Trajectories}

We consider the same $m=1, \hbar=1, a=1$, and $k=15.2$ for trajectories that we used for investigating reduced action. The initial trajectory for examination leaves the upper secondary source with the prolate spheroidal constant of motion $\eta_{a}=-\sin (\pi / 18)$, which has been chosen to be explicitly negative so that the secondary point source and $\eta_{a}$ are in opposite hemispheres with regard to the sign of $\eta$. The corresponding cylindrical constant of the motion is with $\hbar k_{z}=-\hbar k \sin (\pi / 18)$. The trajectory equations, Eqs. (6) and (7), are implicit and solved numerically by the secant method in cylindrical and prolate spheroidal coordinates respectively. The trajectories in prolate spheroidal coordinates are a monotonic function of $\eta$ while in cylindrical coordinates neither $\rho$ nor $z$ are the trajectories monotonic. Hence, the numerical process was better behaved in prolate spheroidal coordinates with regard to convergence by the secant method for successive points of the trajectory. The resulting trajectory transits between the two secondary point sources as exhibited in Fig. 5. For this reason, this trajectory is called a "confined" trajectory. As the double point source experiment has azimuthal symmetry, Fig. 5 exhibits the trajectory projected onto the $\rho, z$-plane in cylindrical coordinates. Figure 5 is presented in cylindrical coordinates to accommodate a change of scale by a factor of ten in $\rho$ at $\rho=0.1$ to facilitate exposition of significant detail and the entire trajectory between the two secondary point sources. Nevertheless, prolate spherical coordinates still renders better insight when examining the trajectory presented on Fig. 5.

Figure 5 is misleading as the apparent symmetry of the trajectory about the $\eta=0$ or $z=0$ plane in Euclidean 3 -space is spurious. In prolate spheroidal coordinates, $\xi \geq 1$ while in cylindrical coordinates $\rho \geq 0$. The point on the trajectory at the origin $(\xi, \eta)=(1,0)$ or $(\rho, z)=(0,0)$, is an inflexion point in Euclidean 3 -space. The trajectory mimics a cubic equation in the neighborhood of the origin where the symmetric pair of constant reduced action contours osculate with zero curvature. As the trajectory transits $z=0, \phi$ 
changes value by $\pm \pi$. This inflexion point of the trajectory in Euclidean 3-space at the coordinate origin $(\rho, z)=(0,0)$ induces the trajectory to be antisymmetric in Euclidean 3-space about the plane $\eta=0$ or $z=0$. The trajectories intersect the contours of constant reduced action in opposite direction in the two hemispheres.

The trajectory, as exhibited by Fig. 5, has turning points in $\xi$ in the vicinity of $k a \eta= \pm 2 \pi, \pm 4 \pi$ or in the vicinity of $\eta= \pm 0.413, \pm 0.827$ or $z= \pm 0.207, \pm 0.413$. At these points, the interference between $\psi_{1}$ and $\psi_{2}$ reinforce each other. This is analogous to the lower turning points for the example considered in the companion paper. ${ }^{3}$ Other turning points in $\xi$ occur for some $\xi>1$ in the vicinity of $k a \eta= \pm \pi, \pm 3 \pi$ or in the vicinity of the hyperboloids $\eta= \pm 0.207, \pm 0.620$ where the interference between $\psi_{1}$ and $\psi_{2}$ oppose each other as the $\cos (\mathrm{ka \eta})$ term in Eq. (7) is the super preponderate cause of destructive interference. These turning points are analogous to the upper turning points for the example considered in the companion paper. (3)

There remains two turning points in $\eta$, which manifest local maximum destructive interference between $\psi_{1}$ and $\psi_{2}$, on Fig. 5 that are located approximately at points $(\xi, \eta) \approx(1.0065, \pm 0.954)$ or $(\rho, z) \approx$ $(0.0171, \pm 0.480)$. Here, the contribution of the factor $\cos (\mathrm{ka \eta})$ in Eq. (7) contributing to self-interference is no longer super preponderate contribution near a secondary source point $(\xi, \eta)=(1, \pm 1)$. The choice of $k a \neq 2 n \pi, n=1,2,3, \cdots$ preempts the existence of latent next "regular" turning point at $k a \eta= \pm 5 \pi$ for such $|\eta| \not \leq 1$ would be nonphysical. Nevertheless, $\psi_{d}$, which has no self-interference at either secondary point source, immediately acquires self-interference upon sortieing from either secondary point source that increases to leading order as $(\xi-\eta)(\xi+\eta)$ in the vicinity of the secondary point sources that in turn induces these "irregular" turning points in conjunction with the behavior of $\cos (\mathrm{ka \eta})$ factor in Eq. (7).

Let us now investigate a set of selected "confined" trajectories that leave the upper secondary point source with various values of $\eta_{a} \leq 0$ so that the secondary source and $\eta_{a}$ are in opposite hemispheres . Figure 6 exhibits the set of selected trajectories whose constants of the motion are given by

$$
\eta_{a}=-\sin (0),-\sin (\pi / 8),-\sin (\pi / 4),-\sin (3 \pi / 8) \approx-0,-0.383,-0.707,-0.924,
$$

where -0 denotes that the limit $\eta_{a} \rightarrow 0$ is from below. By symmetry, Fig. 6 need exhibit only the right upper quadrant. A fifth trajectory for constant of the motion, $\eta_{a}=-\sin (\pi / 2)=-1$ superimposes line $\xi=1$ on Fig. 6 in the range $0 \leq \eta \leq 1$. The trajectories for $\eta_{a} \approx-0.383,-0.707,-0.924$ cross at their mutual inflexion point in Euclidean 3-space at the origin $(\xi, \eta)=(1,0)$. For $\eta_{a}<0$, in the limit that the constant of the motion $\eta_{a} \rightarrow 0$ from below, then its trajectory too goes through the origin and crosses those other trajectories with $\eta_{a}<0$ there. Thus, the origin, $(1,0)$, is a focus for "confined" trajectories.

The trajectories are mutually tangent at the turning points at $\xi=1$ and $k a \eta=2 \pi, 4 \pi$ where there is maximum reinforcement between $\psi_{1}$ and $\psi_{2}$. The trajectories have common turning points at $(\xi, \eta)=$ $(1,2 \pi / k a),(1,4 \pi / k a) \approx(1,0.413),(1,0.827)$ that form foci of the "confined" trajectories on the ellipsoidal principal axis $\xi=1$ The trajectories also do not cross at these foci.

The trajectories have "regular" turning points, where the values of $\xi$ attain local maxima, near the hyperboloids $\eta=\pi / k a, 3 \pi / k a \approx 0.207,0.620$. These "regular" turning points are located at local maxima in the destructive interference between $\psi_{1}$ and $\psi_{2}$. Due to the scale of Fig. 6, only the "regular" turning point manifesting maximum destructive interference for the trajectory with constant of the motion $\eta_{a}=$ $-\sin (3 \pi / 8) \approx-0.917$ is exhibited on Fig. 6 near the unexhibited hyperboloid $\eta=3 \pi / k a \approx 0.620$. For the selected family of exhibited trajectories, the other "regular" turning points of maximum destructive interference are displaced well off the scale of Fig. 6.

The "irregular" turning points exist on Fig. 6 where the trajectories attain $\xi$ values of local maxima for $3 \pi / k a \eta<1$. These "irregular" turning points have values of $\eta$ between approximately 0.955 and 0.975 and increase as the constant of the motion, $\eta_{a}$ increases.

A trajectory in Fig. 6 passes through an alternating series of turning points. The turning points where maximum destructive interference in $\psi_{d}$ occur are interspersed with turning points (foci) where maximum reinforcement occurs. This alternating series of turning points is reminiscent of the companion paper 3 , where alternating turning points manifest creation and annihilation of trajectories. At the turning points of maximum destructive interference one trajectory segment in forward motion merges with a retrograde 
trajectory for mutual annihilation while foci create a forward and retrograde trajectory segments. Thus, the trajectories of Fig. 6 have pattern of alternating forward and retrograde segments with respect to the ellipsoidal coordinate $\xi$ (in Section 3.3 the retrograde motion is shown to be also with respect to time).

Let us now investigate a set of selected trajectories with various values of $\eta_{a} \geq 0$ so that the secondary point source and $\eta_{a}$ are in the same hemisphere with regard to the sign of $\eta$. We first examine the trajectory that leaves the upper secondary source with the spheroidal constant of the motion $\eta_{a}=\sin (\pi / 32) \approx 0.0980$. The corresponding cylindrical constant of motion $\hbar k_{z}=\hbar k \sin (\pi / 32) \approx 15.12681 \hbar$. This trajectory is exhibited on Fig. 7 and is monotonically decreasing in $\eta$ as it asymptotically approaches its constant of the motion $\eta_{a}$ where $\xi$ increases without bound. As such, this trajectory is called "free". The trajectory exhibits turning points for local extrema in the value of $\xi$. The two turning points at local minima of $\xi$ manifesting maximum reinforcement are located near the unexhibited hyperboloids $\eta=2 \pi / k a, 4 \pi / k a$ with values of $\xi>1$. In contradistinction to the "confined" trajectories, "free" trajectories do not have foci on the ellipsoidal principal axis $\xi=1$. The trajectory has turning points at local maxima of $\xi$ near the unexhibited hyperboloids $\eta=\pi / k a, 3 \pi / k a$ that are well displaced off Fig. 7. The trajectory has an additional turning point near $(\xi, \eta)=(3.409,0.128)$ after which the trajectory increases in $\xi$ without bound and sharing the mutual asymptote with the hyperboloid $\eta=\sin (\pi / 32)$.

Figure 8 exhibits the set of four selected "free" trajectories exhibited whose constants of the motion are given by

$$
\eta_{a}=+\sin (0),+\sin (\pi / 8),+\sin (\pi / 4),+\sin (3 \pi / 8) \approx+0,+0.383,+0.707,+0.924
$$

where +0 denotes that the limit $\eta_{a} \rightarrow 0$ is from above. The secondary source and $\eta_{a}$ are in the same hemisphere for these trajectories. The trajectories exhibited on Fig. 8 have positive values of $\eta_{a}$ while those for Fig. 6 have negative. By symmetry, Fig. 8 need exhibit only the right upper quadrant. A fifth trajectory with the constant of the motion, $\eta_{a}=+1$ superimposes on the line $\eta=1$ on Fig. 8 in the range $1 \leq \xi$, that is the the $z$-axis above the upper secondary point source in cylindrical coordinates. All trajectories have monotonically decreasing $\eta$ 's except for the one specified by $\eta_{a}=+1$ as previously discussed. Each trajectory asymptotically approaches its constant of the motion, $\eta_{a}$. Trajectories with constant of the motion $\eta_{a}>3 \pi / k$, that, for the trajectories exhibited on Fig. 8 , includes the two trajectories with $\eta_{a} \approx+0.924,+0.707$ respectively, do not ever reach the value of $\eta \approx+0.620$ where $\cos (k a \eta)=-1$ in Eq. (7) manifesting latent maximum interference. This explains why the behavior of the "free" trajectories become smoother with increasing $\left|\eta_{a}\right|$, (also true for "confined" trajectories where in the limit $\eta_{a} \rightarrow|1|$ the confined trajectory goes to the ellipsoidal principal axis, $\xi=1$ ). The trajectory with $\eta_{a}=+0$ at $\eta=0$ must now be examined in the limit that $\eta_{a} \rightarrow 0$ from above. The trajectory with constant of the motion $\eta_{a}=\sin (\pi / 32)$ as exhibited on Fig. 7 has, as noted in the previous paragraph, a turning point near $(\xi, \eta)=(3.409,0.128)$ after which it proceeds asymptotically to its $\eta_{a}$. This tuning point moves to the origin $(\xi, \eta)=(1,0)$ as $\eta_{a} \rightarrow 0$ from above, and the trajectory for $\eta_{a}=0$ then superimposes on the line $\eta=0$ for $\xi \geq 1$ on Fig. 8. Note that the behavior of the trajectory for in the limit $\eta_{a} \rightarrow 0$ depends on whether the limit is approached from above (i.e., $\eta_{a}$ positive) or below (i.e., $\eta_{a}$ negative) where it was shown earlier in conjunction with Fig. 6 that in the limit $\eta_{a} \rightarrow 0$ from below induces the trajectory not to have a turning point at the origin. The different behaviors for the trajectories for $\eta_{a}= \pm 0$ is the reason for making explicit whether the limit $\eta_{a} \rightarrow 0$ is taken from above or below. The trajectories with $\eta_{a}=+0,-0$ superimpose on each other for the segment between between $(\xi, \eta)=(1,1)$ and $(\xi, \eta)=(1,0)$, but differ beyond the origin. The trajectory for $\eta_{a}=+0$ then propagates out as a straight line, $(\xi, \eta)=(\xi \geq 1,0)$. On the other hand, the trajectory for $\eta_{a}=-0$ proceeds antisymmetrically, as previously noted, to the lower secondary point source, $(\xi, \eta)=(1,-1)$. For completeness, there is a trajectory originating from the lower secondary point source that is the symmetric equivalent to the trajectory originating from the upper secondary point source with $\eta_{a}=+0$.

By Fig. 6 the set of all "confined" trajectories originating from the upper secondary point source with a constant of the motion $\eta_{a}<0$ lie on one side by the trajectory for $\eta_{a}=-0$. By Fig. 8 the set of all "free" trajectories originating from the upper turning point with a constant of the motion $\eta_{a}>0$ lie on the other side by the trajectory for $\eta_{a}=+0$. By symmetry, an analogous situation occurs for trajectories originating 
from the lower secondary point source except that the trajectories with $\eta_{a}>0$ are "confined" while those with $\eta_{a}<0$ are "free". A "free" trajectory has its secondary point source and constant of the motion, $\eta_{a}$ in the same hemisphere with regard to the sign of $\eta$; a "confined" trajectory, opposite hemispheres. The particular case of $\eta_{a}=0$ depends on how $\lim _{\eta_{a} \rightarrow 0}$ is taken. The set of all "confined" trajectories for which $\eta_{a} \neq 0$ form an open domain in $\xi, \eta$-plane that is bounded by the trajectory originating from the upper secondary point source with for $\eta_{a}=-0$ The set of all "free" trajectories originating from either secondary source combined with the trajectory $\eta_{a}=+0$ originating from the upper secondary source and its symmetric equivalent from the lower secondary source form a closed domain in the $\xi, \eta$-plane that is the compliment in the $\xi, \eta$-plane of the open domain formed by the open set of all "confined" domains. Revolving the $\xi, \eta$-plane azimuthally through $2 \pi$ in $\phi$ shows that the trajectories for a quantum dispherical particle span Euclidean 3 -space.

\subsection{Loci of Transit Times}

We consider the same $m=1, \hbar=1, a=1$, and $k=15.2$ that we used for investigating reduced action and trajectories for investigating the loci of transit times for transits in the near region to assist resolving welcher Weg. We have computed from Eqs. (8) and (9) and exhibited on Fig. 9 for the dispherical particle the loci of transit times for $t=0,0.002,0.02,0.04,0.06$. The loci for $t=0$ are points on Fig. 9 on the principal ellipsoidal axis $(\xi=1)$ at $\eta=0,2 \pi / k a, 4 \pi / k a, 1$ or $\eta \approx 0,0.413,0.827,1$. The two coherent secondary sources at $(1, \pm 1)$ nonlocally induce coherent tertiary focal points approximately at $(1,0),(1, \pm 0.413)$ and $(1, \pm 0.827)$ which are focal points of the "confined" trajectories as exhibited by Fig. 6 . The crossing or tangency of trajectories at these induced foci at the same time by Figs. 6 and 9 in the trajectory representation is, in contrast, forbidden in Bohmian mechanics. $14[15]$.

Comparing Figs. 5 through 8 with Fig. 9, one sees that certain trajectories transverse across some loci of transit time many times - in alternating forward or retrograde motion for a particular trajectory. These multiple crossings imply that a particle may be simultaneously at multiple locations manifesting strong nonlocality. Thus the self-entangled dispherical particle ia not necessarily a point particle, and its trajectory is that for a distributed particle.

For the "confined" trajectories, while the series of trajectory segments that alternate forward and retrograde motion render nil transit times from the originating secondary source to the induced tertiary focal points, $t=0$, the transit time from the originating secondary source to a non-focal point on the trajectory in the same hemisphere of the originating secondary source is never negative, i.e., $t \nless 0$; but for opposite hemispheres, never positive, $t \ngtr 0$. On the other hand for "free" trajectories, $t>0$.

We note that the loci for transit times exhibit piecewise separation on Fig. 9. The cut between $\eta=1$ and $\eta=4 \pi / k a \approx 0.827$ in the locus for $t=0.002$ has closed for the locus for time $t=0.02$. The cut centered at $\eta=3 \pi / k a \approx 0.620$ for loci for times $t=0.002,0.02$ have closed for the locus for time $t=0.04$. The cut at $\eta=\pi / k a \approx 0.207$ would close for about transit times $t>0.156$, which are not exhibited on Fig. 9 .

The equation of motion, Eq. (8) is antisymmetric with regard to $\eta$. Physically, a "confined" trajectory, which has an inflexion point in Euclidean 3-space at the origin with the azimuthal coordinate $\phi$ changed by $\pm \pi$ so that it transits the contours (disjointed and otherwise) of constant reduced action in the upper and lower hemispheres in opposite directions. What is either forward or retrograde motion in one hemisphere becomes reversed in the other hemisphere for "confined" trajectories. To generalize, the time of transit for any "confined" trajectories between $\left(\xi_{0}, \eta_{0}\right)$ and $\left(\xi_{0},-\eta_{0}\right)$ is nil. It follows that the quantum dispherical particle has the same transit time from either secondary point source to any other point on the "confined" trajectory.

Contrasting Figs. 1, 2 and 4 with Fig. 9, one sees that shapes and connectivities of the contours of reduced action and loci of transit times do not mimic each other in the near region. Note that the induced tertiary sources are neither intuitively manifested by the contours for reduced action in Figs. 1, 2 and 4 nor obvious in Eq. (4).

After a "confined" trajectory passes through the origin, it then, by the symmetry of Fig. 1, transits the contours of constant reduced action in a reverse direction from the direction that it had transited the 
symmetrically corresponding contours before passing through the origin (that is a "confined" trajectory transits contours of constant $W_{d}$ and the loci of transit time in one hemisphere in the reverse order for which it transited them in the other hemisphere). Since the origin in Euclidean 3-space is an inflexion point and not a turning point for a "confined" trajectory as previously noted, those trajectory segments of the transit between the upper secondary point source $(\xi, \eta)=(1,1)$ and the origin $(\xi, \eta)=(1,0)$ that are in the forward direction become retrograde with regard to time in the transit from the origin $(\xi, \eta)=(1,0)$ and the lower secondary point source $(\xi, \eta)=(1,-1)$ while those segments that are retrograde between the upper secondary point source and origin become forward between the origin and lower secondary point source.

\section{WELCHER WEG}

The secondary point sources of the trajectories for the quantum Young's diffraction are the ellipsoidal focus points for prolate spheroidal coordinate system, which are regular singular point in $\xi$ and $\eta$. 13. The trajectory equation, Eq. (7) has branch point singularities in $\xi$ and $\eta$ at these particular focus (secondary source) points. A trajectory for a self-entangled dispherical particle beginning from from the lower secondary point source, $(\xi, \eta)=(1,-1)$, with constant of the motion $0<\eta_{a} \leq 1$ propagates as a "confined" trajectory with monotonically increasing $\eta$ from an initial value of -1 until it reaches the value of +1 at the upper secondary point source $(\xi, \eta)=(1,1)$, with nil transit time as previously shown. At the upper secondary point source, the "confined" trajectory transitions while rounding the branch point singularity at $(\xi, \eta)=(1,1)$ to become a "free" trajectory with the very same constant of the motion $\eta_{a}$. This transition is accompanied by a change in the azimuthal coordinate $\phi$ of $\pm \pi$. Along the "free" segment of the trajectory, $\eta$ now monotonically decreases from +1 to asymptotically approaching the value of the trajectory's constant of the motion, $\eta_{a}$. Analogously, any "free" trajectory of the upper hemisphere may be coupled at $(\xi, \eta)=(1,1)$ to the corresponding "confined" trajectory with the same constant of the motion, $\eta_{a}$. An analogous situation exists for constants of the motion $-1 \leq \eta_{a}<0$ but with the roles of the upper and lower secondary point sources reversed.

Welcher Weg? Both ways concurrently. The trajectory for the quantum dispherical particle through any point in space will have at least a "confined" segment whose terminals are the two coherent secondary point sources. The time of transit for the quantum dispherical particle from either secondary point source to any point on its trajectory will be the same. As previously shown, the "confined" and "free" trajectories span Euclidean 3- space.

Perhaps, quantum mechanics for over eight decades had been asking the wrong question, "Welcher Weg?" It should have been asking, "How both ways simultaneously?"

\section{APPENDIX. QUANTUM ERASURE}

Let us consider a hypothetical experiment for a quantum particle that is half Young's diffraction and half Lloyd's mirror. We combine these two experiments out of phase in such a manner to swap quantum information on self-entangled dispherical wave function for quantum information on spherical wave functions. Such a swapping implies a quantum erasure. Herein, we are not studying the phenomenon of the quantum erasure - merely applying it as a confidence building measure to demonstrate that a spherical wave function can be synthesized from two dispherical wave functions and substantiate that nonlocal entangled quantum particles represented by dispherical wave functions are physical.

Let us modify our hypothetical experiment of interference between two coherent secondary point sources. We still consider the behavior of a solitary quantum particle. The first modification to Young's diffraction experiment is the interference between two secondary point sources is still coherent but now anti-correlated, that is the resultant quantum dispherical wave function $\psi_{Y}$ for Young's diffraction is now given as $\psi_{Y}=$ $\psi_{1}-\psi_{2}$. A sole primary source emits a solitary quantum particle with a specified de Broglie wavelength that after going through an initial 50:50 splitter actuates the secondary point sources 1 and 2. Secondary point source 1 is arbitrarily made the lower point source; secondary point source 2 , the upper secondary point 
source. Anti-correlation is achieved by making the path length from the primary source to lower secondary point source $N$ de Broglie wavelengths while inserting a second 50:50 splitter that in turn splits the path from the primary source to the upper secondary point source into two branches. One branch is $N-1 / 2$ de Broglie wavelengths long; the other, $N+1 / 2$ de Broglie wave lengths. Having two branches to the upper secondary source confounds a time-of-arrival analysis for determining welcher Weg for a solitary quantum particle.

The next modification is to insert a half-silvered mirror along the the $\eta=0$ plane. This produces two partial Lloyd's mirror experiments: one for the lower secondary point source for $-1 \leq \eta \leq 0$; the other for the upper secondary point source for $0<\eta<+1$. Concurrently the half-silvered mirror posits the virtual point source for each Lloyd's mirror at the alternate secondary point source. Hence, the dispherical wave function for Lloyd's mirror in the lower infinite hemisphere is given by $\psi_{L}=+\left(\psi_{1}+\psi_{2}\right) / 2^{1 / 2},-1 \leq \eta \leq 0$ and in the upper hemisphere is given by $\psi_{L}=-\left(\psi_{1}+\psi_{2}\right) / 2^{1 / 2},-1 \leq \eta \leq 0$.

The half-silvered mirror also reduces the amplitude of dispherical wave function for Young's diffraction by the factor $2^{-1 / 2}$ so that $\psi_{Y}=+\left(\psi_{1}-\psi_{2}\right) / 2^{1 / 2}$ throughout all space. There exists interference between the two dispherical waves, $\psi_{Y}$ and $\psi_{L}$. These two dispherical waves can be summed as

$$
\psi_{Y}+\psi_{L}=\left\{\begin{array}{cc}
2^{1 / 2} \psi_{1}, & -1 \leq \eta \leq 0 \\
-2^{1 / 2} \psi_{2}, & 0 \leq \eta \leq+1
\end{array}\right.
$$

Hence, a spherical wave can be synthesized from two dispherical waves. Cascaded entanglement recovers the spherical wave. Both the spherical and dispherical waves are wave functions of the Schrödinger equation. It is just as valid to work with dispherical wave functions as it is to work with spherical waves by the superpositional principle of linear homogeneous differential equations.

\section{Acknowledgements}

I heartily thank Marco Matone for his interesting discussions. I also thank Robert Carroll, Alon E. Faraggi, Bill Poirier and Robert E. Wyatt for their contributions and encouragement.

\section{References}

1. E. R. Floyd, Phys. Rev. D 34, 3246 (1986).

2. E. R. Floyd, Gravitation and Cosmology: From the Hubble Radius to the Planck Scale; Proceedings of a Symposium in Honour of the $80^{\text {th }}$ Birthday of Jean-Pierre Vigier, ed. by R. L. Amoroso, G. Hunter, M. Kafatos and J.-P. Vigier, (Kluwer Academic, Dordrecht, 2002), extended version promulgated as quant-ph/00009070.

3. E. R. Floyd, Found. Phys. 37, 1386 (2007), quant-ph/0605120.

4. A. E. Faraggi and M. Matone, Int. J. Mod. Phys. A 15, 1869 (2000), hep-th/98090127.

5. G. Bertoldi, A. E. Faraggi and M. Matone, Class. Quant. Grav. 173965 (2000), hep-th/9909201

6. R. Carroll, Can. J. Phys. 77, 319 (1999), quant-ph/9904081 Quantum Theory, Deformation and Integrability (Esevier, 2000, Amsterdam) pp. 50-56; Uncertainty, Trajectories, and Duality, quant-ph/0309023.

7. P. M. Morse and H. Feshbach, Methods of Theoretical Physics, Part II (McGraw-hill, New York, 1953), p. 1284 .

8. C. Philippidis, C. Dewdney and B. J. Hiley, Neuvo Cimento 52B, 15 (1979).

9. R. Guantes, A. S. Sanz, J. Margalef-Roig and S. Miret-Artés. Surf. Sci. Rep. 53199 (2004).

10. D. Bohm, Phys. Rev. 85166 (1953).

11. E. R. Floyd, Phys. Rev. D 26, 1339 (1982).

12. H. Goldstein, Classical Mechanics $2^{\text {nd }}$ ed. (Addison-Wesley, Reading, 1980) p. 441.

13. P. M. Morse and H. Feshbach, loc. cit. Part I, p. 661.

14. P. R. Holland, the Quantum Theory of Motion (Cambridge, Cambridge, 1993) pp. 85-86, 183, 201.

15. Y. Zhao and N. Makri, J. Chem. Phys. 119, 60 (2003) 


\section{Figure Captions}

Fig. 1. Contours of constant reduced action for $W_{d}=0.5 h, 1 h, 1.5 h, \cdots, 5 h$ for the quantum dispherical particle with $m=1, \hbar=1, a=1$ and $k=15.2$.

Fig. 2. Contours of constant reduced action for the quantum dispherical particle as solid lines. The dashed lines are the projection onto the $\xi, \eta$-plane of the hyperboloids $\eta=0.207,0.620$ near where maximum destructive interference occurs between $\psi_{1}$ and $\psi_{2}$.

Fig. 3. Contours of constant reduced action for $W_{d}=4 h, 5 h, 6 h$ for the quantum dispherical particle with $m=1, \hbar=1, a=1$ and $k=24.3$. The dashed lines are the projection onto the $\xi, \eta$-plane of the hyperboloids $\eta=0.129,0.388,0.646,0.905$ near where maximum destructive interference occurs between $\psi_{1}$ and $\psi_{2}$.

Fig. 4. Contours of constant reduced action for $W_{d}=1.315815 h, 1.316815 h, 1.317815 h$ in the vicinity of the origin for the quantum dispherical particle with $m=1, \hbar=1, a=1$ and $k=15.2$.

Fig. 5. Trajectory for the quantum dispherical particle originating from the upper secondary source with constant of the motion $\eta_{a}=-\sin (\pi / 18)$. Note change of scale in $\rho$ by a factor of ten at $\rho=0.1$, denoted by the dashed vertical line, to facilitate exposition.

Fig. 6. A set of selected "confined" trajectories for the quantum dispherical particle from the upper secondary source for the constants of the motion $\eta_{a} \approx-0,-0.383,-0.707,-0.924$. A fifth trajectory for $\eta_{a}=-1$ superimposes upon the line $\xi=1$ in the range $0 \leq \eta \leq 1$.

Fig. 7. The trajectory for the quantum dispherical particle originating from the upper secondary source with constant of the motion $\eta_{a}=+\sin (\pi / 32)$.

Fig. 8. A set of selected "free" trajectories for the quantum dispherical particle from the upper secondary source for the constants of the motion $\eta_{a} \approx+0,+0.383,+0.707,+0.924$. A fifth trajectory for $\eta_{a}=+1$ superimposes upon the line $\eta=1$ in the range $1 \leq \xi$.

Fig. 9. Loci of selected transit times. At transit time $t=0$., induced tertiary sources are at $(\xi, \eta) \approx$ $(1,0.827),(1,0.413),(1,0)$. 
This figure "Fig1WW.png" is available in "png" format from: http://arxiv.org/ps/quant-ph/0605121v3 
This figure "Fig2WW.png" is available in "png" format from: http://arxiv.org/ps/quant-ph/0605121v3 
This figure "Fig3WW.png" is available in "png" format from: http://arxiv.org/ps/quant-ph/0605121v3 
This figure "Fig4WW.png" is available in "png" format from: http://arxiv.org/ps/quant-ph/0605121v3 
This figure "Fig5WW.png" is available in "png" format from: http://arxiv.org/ps/quant-ph/0605121v3 
This figure "Fig6WW.png" is available in "png" format from: http://arxiv.org/ps/quant-ph/0605121v3 
This figure "Fig7WW.png" is available in "png" format from: http://arxiv.org/ps/quant-ph/0605121v3 
This figure "Fig8WW.png" is available in "png" format from: http://arxiv.org/ps/quant-ph/0605121v3 
This figure "Fig9WW.PNG" is available in "PNG" format from: http://arxiv.org/ps/quant-ph/0605121v3 